\title{
Development of a model to quantify the accessibility of a Canadian trauma system
}

\author{
Gavin Tansley, $\mathrm{MD}, \mathrm{MPH}^{*}$; Nadine Schuurman, $\mathrm{PhD}^{\dagger}$; Mete Erdogan, $\mathrm{PhD}, \mathrm{MHI}^{\ddagger}$; \\ Matthew Bowes, $\mathrm{MD}^{\S}$; Robert Green, $\mathrm{MD}^{\mathbb{I}_{*}^{*}}$; Mark Asbridge, $\mathrm{PhD}^{\dagger+}$; Natalie Yanchar, MD, MSc*
}

\section{ABSTRACT}

Objectives: Trauma systems have been widely implemented across Canada, but access to trauma care remains a challenge for much of the population. This study aims to develop and validate a model to quantify the accessibility of definitive care within one provincial trauma system and identify populations with poor access to trauma care.

Methods: A geographic information system (GIS) was used to generate models of pre-scene and post-scene intervals, respectively. Models were validated using a population-based trauma registry containing data on prehospital time intervals and injury locations for Nova Scotia (NS). Validated models were then applied to describe the population-level accessibility of trauma care for the NS population as well as a cohort of patients injured in motor vehicle collisions (MVCs).

Results: Predicted post-scene intervals were found to be highly correlated with documented post-scene intervals ( $\beta$ 1.05, $p<0.001$ ). Using the model, it was found that $88.1 \%$ and $42.7 \%$ of the population had access to Level III and Level I trauma care within 60 minutes of prehospital time from their residence, respectively. Access for victims of MVCs was lower, with $84.3 \%$ and $29.7 \%$ of the cohort having access to Level III and Level I trauma care within 60 minutes of the location of injury, respectively.

Conclusion: GIS models can be used to identify populations with poor access to care and inform service planning in Canada. Although only $43 \%$ of the provincial population has access to Level I care within 60 minutes, the majority of the population of NS has access to Level III trauma care.

\section{RÉSUMÉ}

Contexte: Différents systèmes de traumatologie ont été mis sur pied un peu partout au Canada, mais l'accès aux centres de traumatologie est difficile pour une grande partie de la population. L'étude décrite ici avait pour buts d'élaborer et de valider un modèle visant à quantifier l'accessibilité à des centres de soins pluridisciplinaires, rattachés à un seul système provincial de traumatologie, et de cerner les populations ayant difficilement accès aux centres de traumatologie.
Méthode: Les chercheurs ont eu recours à un système d'information géographique (SIG) pour générer des modèles d'intervalles d'intervention avant et après événement, respectivement. Les modèles ont été validés à l'aide d'un registre de traumatologie reposant sur la population et contenant des données sur les intervalles d'intervention en phase préhospitalière ainsi que sur les lieux d'accidents en Nouvelle-Écosse (N.-É.). Une fois validés, les modèles ont ensuite été appliqués pour établir le degré d'accessibilité aux centres de traumatologie pour l'ensemble de la population de la N.-É. ainsi que pour une cohorte de personnes ayant subi des blessures dans des accidents de véhicules automobiles (AVA).

Résultats: Une forte corrélation a été établie entre les intervalles d'intervention prévus après un événement et les intervalles $d$ 'intervention documentés après un événement $(\beta=1,05 ; p<0,001)$. $D^{\prime}$ après le modèle, $88,1 \%$ et $42,7 \%$ de la population avaient accès, en phase préhospitalière, à un centre de traumatologie de niveau III ou de niveau I en I'espace de 60 minutes depuis leur lieu d'habitation, respectivement. Par contre, $84,3 \%$ et $29,7 \%$ des personnes blessées dans des AVA, soit des taux moins élevés que dans le groupe précédent, ont eu accès à un centre de traumatologie de niveau III ou de niveau I en I'espace de 60 minutes depuis le lieu de l'accident, respectivement.

Conclusions: Les modèles reposant sur les SIG peuvent servir à cerner les populations ayant difficilement accès aux centres de traumatologie, et à guider la planification des services au Canada. Bien que $43 \%$ seulement de la population en N.-É. aient accès à un centre de traumatologie de niveau 1 en l'espace de 60 minutes, la grande partie de la population de la province a accès un centre de traumatologie de niveau III.

Keywords: trauma, access, GIS, Geographic Information Systems

\section{INTRODUCTION}

Injury is a major cause of mortality and health expenditure in Canada, accounting for over 15,000 deaths and

From the *Department of Surgery, ๆDepartment of Critical Care Medicine, **Department of Emergency Medicine, and the $\dagger+D e p a r t m e n t$ of Community Health and Epidemiology, Dalhousie University, Halifax, NS; tDepartment of Geography, Simon Fraser University, Burnaby, BC; ¥Trauma Nova Scotia, Halifax, NS; and the §Nova Scotia Medical Examiner Service, Dartmouth, NS.

Correspondence to: Dr. Gavin Tansley, Dalhousie University, 5850 University Ave., Halifax, NS B3K 6R8; Email: gtansley@dal.ca 
\$20 billion of direct and indirect costs annually. Although the landscape of Canadian trauma care has evolved substantially over recent years, it maintains adherence, like all medical services, to the guiding principles of the Canada Health Act (public administration, comprehensiveness, universality, portability, and accessibility). ${ }^{2}$ Therefore, access to urgent and appropriate essential care is not only an expectation, but also mandated by law for all Canadian citizens and landed immigrants.

Trauma care should be provided at an accredited trauma centre (TC) with the resources and personnel required to effectively assess severely injured patients and provide definitive care. ${ }^{3,4}$ Within a trauma system, TCs are often tiered. Generally, Level I TCs have leadership roles in the provincial trauma system and provide most of the major trauma care and specialized trauma services for the province, in addition to trauma training and research programs. Level II TCs are needed in jurisdictions without a Level I TC or where the major trauma caseload is too large for a single Level I TC to manage. Level III TCs are required in smaller urban or rural communities without timely access to Level I or Level II TCs. In Nova Scotia (NS), two Level I TCs in Halifax provide the majority of the higher level and definitive trauma care, and eight Level III TCs provide the initial resuscitation and care of severely injured patients with subsequent transfer of patients requiring complex care to the higher level TCs. This kind of trauma care regionalization has resulted in an estimated $15 \%$ decrease in the likelihood of death following injury in North America, and represents one of the major advancements in trauma care in recent decades. ${ }^{5}$ Although this approach helps ensure that finite resources are optimally managed, it has the consequence of concentrating these resources at fewer locations. Because accessing trauma care is required before survival benefits can be realized, measuring accessibility becomes an important component of trauma system evaluation and equitable health care delivery in Canada. ${ }^{6}$

The geographic component of access to trauma care in the province of NS has been previously studied. ${ }^{7,8}$ However, these studies have been limited strictly to population-level analyses examining access to the Level I TCs and did not incorporate important trauma-related data such as injury location and pre-scene time into their analysis. Without a comprehensive, validated model of trauma care accessibility, populations with poor access to provincial resources cannot be reliably identified. To address the limitations of prior work in this area, the present study aims to develop a model to quantify spatial access to Level I and Level III trauma care in NS, and to validate the model using a provincial database containing a retrospective cohort of patients severely injured in a motor vehicle collision (MVC). By applying this model to the provincial population as well as to the severely injured cohort, the accessibility of trauma care for these two populations is compared.

Because NS trauma care is comparable to many other provincial trauma systems, results of this study will likely be applicable to the broader Canadian context. Identifying areas of poor access to trauma care will allow for effective allocation of trauma care resources in Canada.

\section{METHODS}

\section{Study design}

A geographic information system (GIS)-based analysis was conducted to create and validate a model of prescene and post-scene prehospital times for victims of trauma in NS. This model was created to quantify the accessibility of trauma care for the population of NS as well as a cohort of patients severely injured in MVCs.

\section{Setting}

NS is the second most densely populated province in Canada with an average population density of 18 persons $/ \mathrm{km}^{2}$. Notably, the majority of the population resides outside of the province's only census metropolitan area, resulting in a high prevalence of rural trauma. Trauma care in NS is divided amongst eight Level III TCs, one adult Level I TC, and one pediatric Level I TC. ${ }^{9}$ The Level I TCs are the only facilities in the province with acute neurosurgical capacity. Emergency Health Services (EHS) provides ground-based prehospital transport using a comprehensive network of ground ambulances deployed by a dynamic dispatch system designed to maximize provincial coverage. Although aeromedical transport is also available, these assets are used in fewer than $5 \%$ of scene responses and are therefore not the focus of this study.

\section{Study data}

Injury location and prehospital time interval data were obtained from the Nova Scotia Trauma Registry 
(NSTR), which is a population-based database of all major trauma cases in the province and maintained by the Nova Scotia Trauma Program (NSTP). The NSTP definition of major trauma is any injury with an Injury Severity Score (ISS) $\geq 12$ and an appropriate International Classification of Disease External Cause of Injury Code. The NSTR also included all trauma team activations regardless of ISS, and traumas with an appropriate injury mechanism that resulted in death either at the scene, in the emergency department, or within 24 hours of admission to a Level III or Level I TC. Prescene times were defined as the time interval between EHS activation and arrival on the scene of injury. Postscene times were defined as the time interval between EHS departing the scene of injury and arriving at a designated TC. EHS (or the coroner in cases of scene deaths) records the coordinates of the pickup location of all victims using the Global Positioning System (GPS). These data were abstracted into the NSTR along with prehospital time intervals collected automatically into the patient's electronic record. All injuries related to MVCs captured within the NSTR's major injury data set between January 1, 2005 and December 13, 2013 were eligible for inclusion. Individuals who were missing GPS coordinates or whose pickup location was inconsistent with the injury location were excluded. All duplicate entries were removed prior to analysis.

The provincial road network used in the analyses was obtained from a commercially available data set (CanMap, DMTI Spatial, Markham, ON). The locations of Level I and Level III TCs were also obtained from this data set. Commercially available GIS software (ArcMap, Esri, Redlands, CA) was used for all geographic analyses. Statistical analyses were performed using Stata v14 (StataCorp, College Station, TX).

\section{Cost-distance analysis}

Cost-distance analyses were performed to model travel times from all points in NS to the nearest Level I or Level III TC. These analyses were performed by generating a $100-\mathrm{m}^{2}$ grid over the entire province and assigning a time to each grid square corresponding to the amount of time required to cross it. This time was calculated using the speed limits of roads within the grid square. Cells without a road were assigned a value corresponding to a travel speed of $5 \mathrm{~km} \cdot \mathrm{h}^{-1}$ (i.e., the average speed of walking). An algorithm was then applied to calculate the time required to travel from each $100-\mathrm{m}^{2}$ grid square to the nearest Level I or Level III TC.

\section{Comparing predicted and documented post-scene times}

Estimates from the cost-distance models were validated by comparing these data with recorded post-scene times for a cohort of patients injured in MVCs who were directly transported to a Level I TC from the scene of injury using ground ambulances. Individuals with unknown post-scene intervals or injury locations were excluded. The potential post-scene time for each of these points was identified by extracting the value of the Level I TC cost-distance output associated with the point location of the MVC. The recorded post-scene time of each incident was retrieved from the NSTR. The association between the two intervals was illustrated graphically and analysed statistically using linear regression.

\section{Pre-scene time modelling}

The point locations of all MVCs occurring during the study period were plotted and linked to their corresponding pre-scene intervals retrieved from the NSTR. The Global Moran's I was calculated to determine whether the location where an MVC occurred was associated with the observed pre-scene time. This calculation is a widely used statistical means of detecting relatedness among adjacent points in a study area. ${ }^{10}$ To generate a continuous surface of pre-scene time estimates across the study area, spatial interpolation was performed using the Kriging method. ${ }^{11}$ This method is used to fit a model to geographically related data, which can then be used to estimate values at locations where no data are available (i.e., where no injuries have occurred). Applying this model to the entire study area resulted in a continuous, smoothed, $100-\mathrm{m}^{2}$ grid of pre-scene time estimates for amalgamation with the post-scene time estimates. A Gaussian model provided the best fit to the data, based on assessments of several error parameters. Data with no recorded pre-scene time were excluded.

\section{Estimating the accessibility of trauma care}

A model of total ambulance travel time was created by combining the post-scene time estimates with the 
pre-scene time estimates for each cell in the study area. This model was overlaid on a population layer of census dissemination areas (DAs) to quantify the accessibility of trauma care for the population of NS. Any DAs with an average travel time of $\leq 60$ minutes were identified and expressed as a proportion of the total population of NS. The 60-minute time interval was chosen to be consistent with prior studies, ${ }^{7,12}$ but recognizing this interval is arbitrary; the average travel time for the entire NS population was plotted in a histogram to illustrate the distribution of potential spatial access to trauma care in NS.

Overlaying the point locations of major traumas related to MVCs over the model allowed for estimates of access to trauma care for a cohort of patients captured in the NSTR during the study period. The predicted travel time corresponding to the point location of each injury was extracted from the model outputs and plotted graphically.

\section{RESULTS}

\section{Study data}

Between January 1, 2005 and December 13, 2013, a total of 1,568 trauma patients injured in MVCs were eligible for inclusion. Following the exclusion of duplicates and entries with missing or inconsistent injury locations, 1,304 trauma cases were suitable for geographic analysis (Figure 1).

\section{Cost-distance analysis of post-scene time}

The cost-distance analysis of ground-based travel time to trauma care in the province of NS is illustrated in Figure 2. Expectedly, regional variation in travel time to the Level I TCs is evident, with the majority of the NS land mass farther than 60 minutes of driving time. Level III TCs are more readily accessible, with most points in the province within 60 minutes of driving time to one of these facilities.

\section{Validation of model}

To determine the accuracy of the cost-distance analysis output in predicting post-scene times, the documented post-scene times of all individuals transported directly from the scene to tertiary care were plotted against the post-scene times predicted from the cost-distance

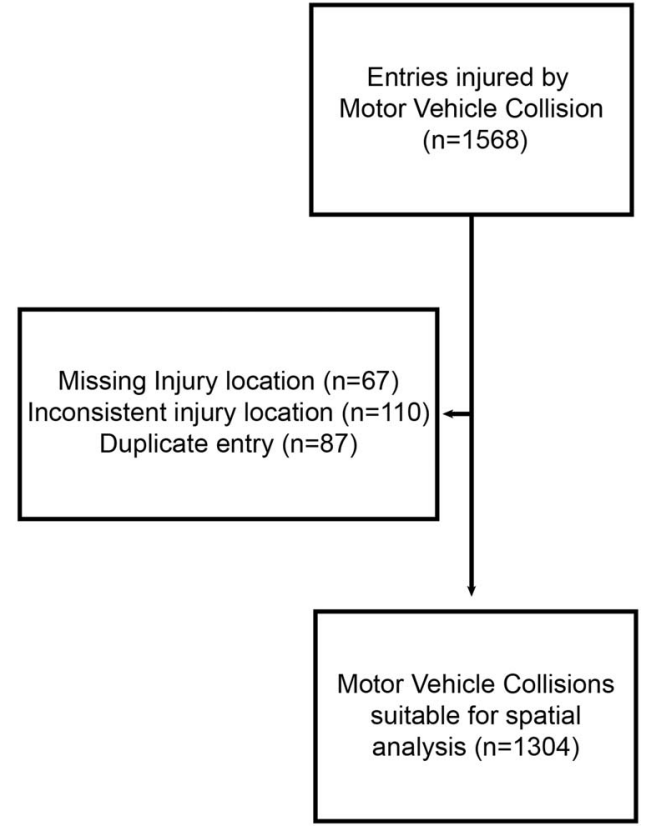

Figure 1. Flow chart of study population.

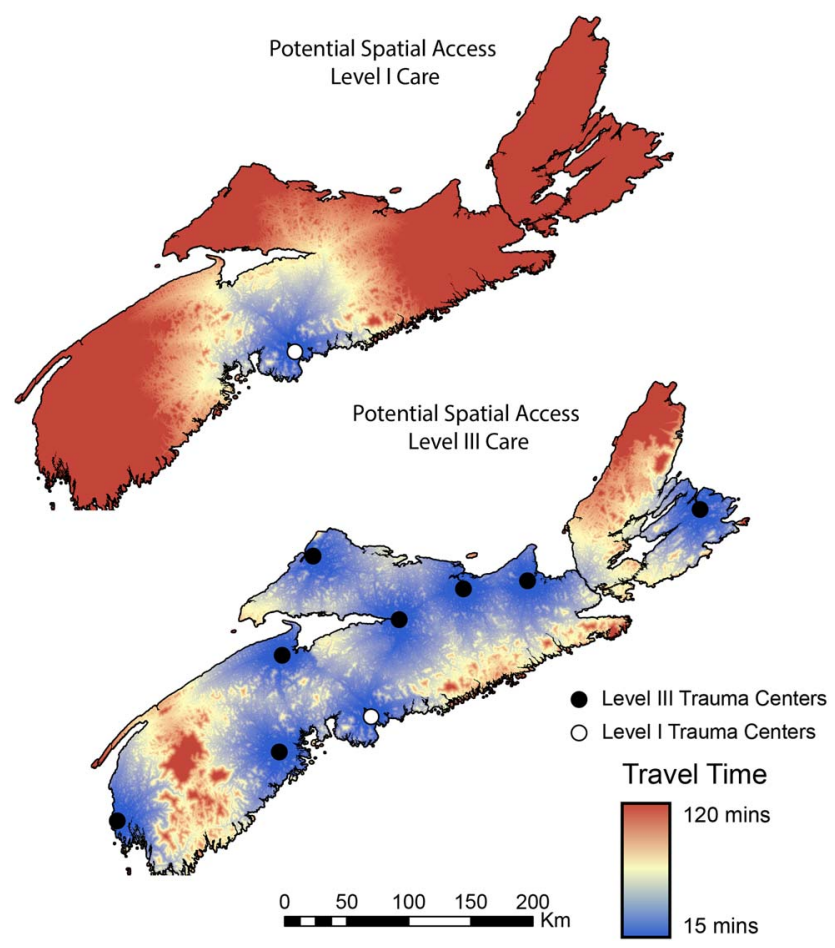

Figure 2. Results of ground-based cost-distance analysis illustrating the predicted driving times to Level III and Level I trauma care for Nova Scotia.

analysis for the same injury location. The results of this comparison are depicted graphically in Figure 3. Following the exclusion of entries with missing data and entries with predicted post-scene times $>60$ minutes, 


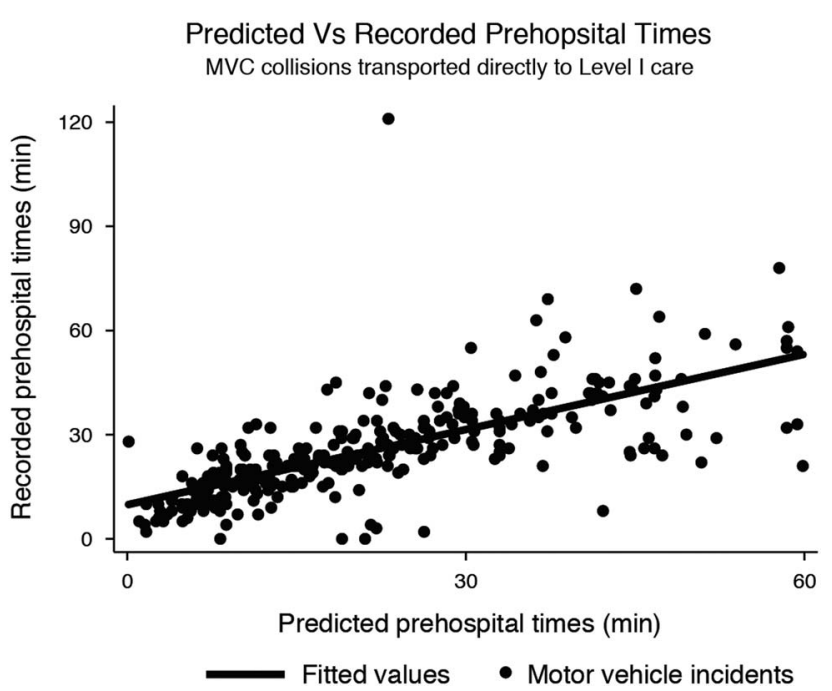

Figure 3. Scatterplot with fitted line depicting the relationship between predicted and recorded post-scene times. The analysis is based on 290 observations in which a victim had a predicted post-scene time of $\leq 60$ minutes and was transported directly from the scene to Level I care.

290 observations remained for analysis. Linear regression ultimately demonstrated a near $1: 1$ relationship between the two time intervals ( $\beta 1.05, p<0.001$, forced intercept of 0 ), supporting the validity of the model for the study population.

\section{Pre-scene time estimations}

Of the 1,304 MVC victims suitable for spatial analysis, pre-scene times were available for 1,222 individuals (93.7\%). There was strong evidence of spatial relatedness within this sample (Moran's Index $0.88, p<0.001$ ). A continuous surface of predicted pre-scene time was created using the Kriging method (Figure 4). This model illustrates the localization of shorter predicted pre-scene intervals around TCs, with longer pre-scene times associated with the more remote areas of the province's interior such as Kejimkujik National Park. The narrow range of predicted pre-scene times between 5 minutes and 35 minutes suggests that the province is uniformly serviced by the EHS.

\section{Access to trauma care in NS}

By combining this pre-scene model with the post-scene model illustrated in Figure 2, a model of total ambulance travel time was generated. By amalgamating this model with a population layer of DAs, it was determined that $88.1 \%$ and $42.7 \%$ of the NS population

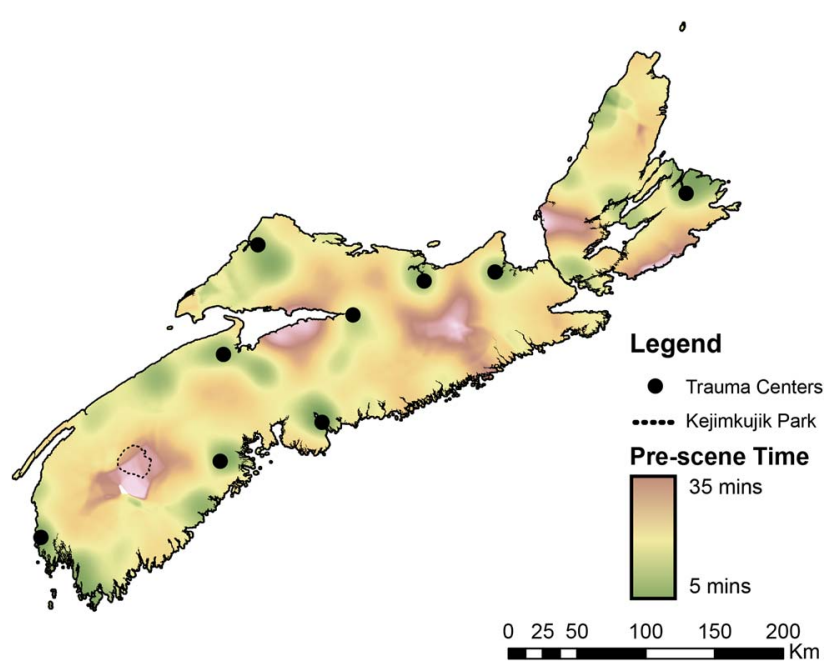

Figure 4. Estimates of pre-scene times in Nova Scotia. Data represent results of a Kriging model based on the documented pre-scene times of 1,304 patients injured in MVCs.

reside within 60 minutes of prehospital time to a Level III TC and Level I TC, respectively (median time to Level III TC: 24.3 minutes; median time to Level I TC: 78.1 minutes). The distributions of trauma care accessibility are illustrated in Figure 5 (A, B).

By plotting the locations of major traumas resulting from MVCs and extracting the ground-based transport time predicted for that location, it was possible to determine the potential spatial access to trauma care for a cohort of injured patients. The results of this analysis were lower compared to the population-level analysis with $84.3 \%$ and $29.7 \%$ of MVC-related injuries occurring within 60 minutes of a Level III TC and Level I TC, respectively (median time to Level III TC: 32.5 minutes; median time to Level I TC: 97.3 minutes). The distributions of predicted travel times are shown in Figure $5(\mathrm{C}, \mathrm{D})$.

\section{DISCUSSION}

This study represents a comprehensive descriptive analysis of the accessibility of trauma care in NS by ground transportation. Using validated GIS-based methods, we were able to demonstrate high population-level access to Level III TCs within 60 minutes of driving time. Population-level access to Level I trauma care was considerably more variable, with $57.3 \%$ of the NS population residing greater than 60 minutes of prehospital time from the provincial 

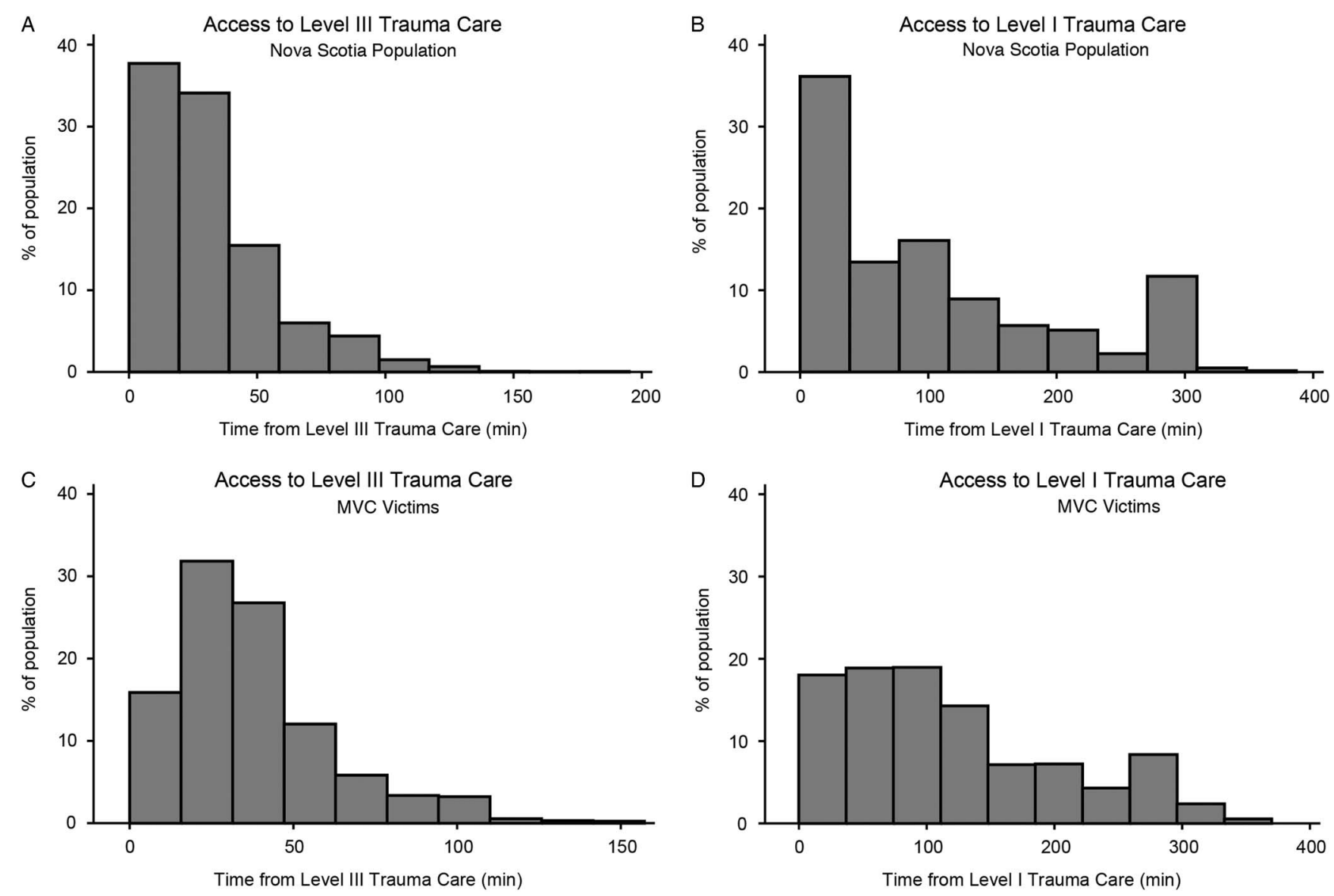

Figure 5. Estimated access to trauma care by ground-based travel in Nova Scotia. A) Access to Level III trauma care for the population of Nova Scotia. B) Access to Level I trauma care for the population of Nova Scotia. C) Access to Level III trauma care for victims of motor vehicle collisions in Nova Scotia. D) Access to Level I trauma care for victims of motor vehicle collisions in Nova Scotia.

Level I adult TC. An analysis of patients with MVCrelated trauma determined that $84.3 \%$ of the cohort was injured within 60-minutes prehospital time of a Level III TC, whereas only $29.7 \%$ were within 60 -minutes prehospital time of a Level I TC. These findings demonstrate that GIS modelling can be a valuable tool for identifying populations with poor access to trauma care.

The geographic component of access to trauma care can be considered at the population level (potential access) or through studying service utilization (revealed access). The first Canadian assessment of access to TCs was a study by Hameed et al., which aimed to quantify the accessibility of all Level I and II TCs nationally using a network analysis method. ${ }^{7}$ This method incorporates road attributes such as speed limits and intersections to arrive at a predicted travel time for a given section of road between a defined start and end point. It has been suggested that this method is one of the preferred GIS-based methods for assessing access because it incorporates barriers such as water bodies and mountain ranges that are ignored in estimates based on straight line distances. ${ }^{13}$ The results of that study demonstrated that $77.5 \%$ of Canadians reside within 1 hour of road travel time to a Level I or II TC. However, estimates for NS were considerably lower than this with only $42 \%$ of the population within 1 hour of driving time to the province's only Level I or II adult TC. ${ }^{7}$ A subsequent study by Lawson et al. attempted to evaluate potential spatial access to trauma care for a Canadian cohort of severely injured patients based on their residential postal codes. ${ }^{8}$ Their results were largely consistent with the findings of Hameed et al., with $41 \%$ of severely injured Nova Scotians residing within 1 hour of driving time to a Level I or II TC.

Although these studies provide useful insights into the spatial accessibility of trauma care in Canada, they 
are not without their limitations. The methods used in these studies relied on the use of speed limits on roads and were not validated for an emergency response system where actual travel speeds may differ. Additionally, using the location of residences as a surrogate for a place of injury assumes that injury is randomly distributed throughout the population and patients get injured at or near their homes. Both of these assumptions have been challenged. ${ }^{14-16}$ Furthermore, the studies by Hameed et al. and Lawson et al. likely represent overestimates of spatial access due to the exclusion of ambulance response times in their analyses. Finally, exclusion of Level III TCs from the study models and the use of arbitrarily defined 60-minute service areas make it difficult for these studies to be applied by policymakers in all areas of Canada.

In the present study, we demonstrated that median predicted prehospital times to the Level I and Level III TCs for a cohort of patients injured in MVCs were $25 \%$ and $33 \%$ longer than estimates based on population distributions, respectively. This is not an unexpected finding given that high-speed roadways are typically located outside of population dense areas, but provides further evidence of the inaccuracies associated with using residence locations to measure access in a trauma system predominated by MVC-related injuries. We also used various GIS modelling techniques to estimate spatial access and predict driving times, which have not been previously applied to evaluations of trauma care access. ${ }^{17-20}$ These methods have the advantage of providing estimates over a continuous surface, allowing for rapid visualization of trends. The dynamic dispatch system used in NS and the lack of availability of ambulance locations at the time of response prevented the use of more traditional routing methods to estimate pre-scene times. Although low data point densities may limit the accuracy of estimates, the high concentration of MVCs in population dense areas allowed for reasonable population-level estimates.

This study is limited by the use of a single trauma system in model development. Although the infrastructure incorporated into the model is comparable nationwide, validation will be required in another setting before the model can be generalized. Additionally, inaccuracies in data reporting could potentially influence the observed results. However, this is made less likely by the quality control measures used by the NSTR and the relative completeness of the data. Finally, this study did not incorporate aeromedical transport into the model. Because this has the potential to substantially impact the accessibility of trauma care for rural populations, future work may aim to describe this impact. However, the utility of these models will be limited by the infrequency of aeromedical transport in most Canadian trauma systems.

Importantly, the population-level results obtained using these methods are largely consistent with the previous work on access to trauma care that was performed in NS. ${ }^{7,8}$ However, without outcomes data for the injured cohort, it is impossible to determine whether spatial access to Level I or Level III TCs influences mortality following injury. Performing an evaluation of spatial access that includes patient outcomes will be important for improving the delivery of trauma care in Canada. Although previous work by Lawson et al. did demonstrate an increased likelihood of death in individuals with poorer access to the Level I TC in NS, the analysis used in this work was not statistically adjusted for relevant confounding variables or based on precise injury locations. ${ }^{8}$ Additionally, because timely access to neurosurgical capacity is known to influence outcomes following traumatic brain injury, ${ }^{21,22}$ and these services are only available in NS at the Level I TCs, evaluating Level I and Level III TCs independently will be important.

\section{CONCLUSIONS}

Validation and application of this model to describe the accessibility of trauma care in NS confirm that there is low potential spatial access to Level I TCs in the province. However, the high accessibility of Level III TCs suggests that these institutions need to play a significant role in NS trauma care. Ongoing maintenance and expansion of the capacity at Level III TCs will be an important component of trauma care improvement in the province. Particular attention needs to be paid to Level III TCs located in areas of the province that have the lowest access to the Level I TCs. Ensuring that Level III TCs have the capacity and resources to provide high-quality emergency care is crucial for ensuring equal access to trauma care in the province. Evaluating the impact of spatial access to Level I and Level III TCs on trauma patient outcomes will be another important component of ongoing research.

Competing interests: None declared. 


\section{REFERENCES}

1. Billette J-M, Janz T. Injuries in Canada: insights from the Canadian Community Health Survey; 2011. http://www. statcan.gc.ca/pub/82-624-x/2011001/article/11506-eng.htm.

2. Canada Health Act (R.S.C., 1985, c. C-6). Available at: http://laws-lois.justice.gc.ca/eng/acts/c-6/.

3. Liberman M, Mulder DS, Jurkovich GJ, et al. The association between trauma system and trauma center components and outcome in a mature regionalized trauma system. Surgery 2005;137:647-58; doi:10.1016/j.surg.2005.03.011.

4. MacKenzie EJ, Rivara FP, Jurkovich GJ, et al. A national evaluation of the effect of trauma-center care on mortality. $N$ Engl 7 Med 2006;354:366-78; doi:10.1056/NEJMsa052049.

5. Celso B, Tepas J, Langland-Orban B, et al. A systematic review and meta-analysis comparing outcome of severely injured patients treated in trauma centers following the establishment of trauma systems. 7 Trauma 2006;60:371-8; discussion 378, doi:10.1097/01.ta.0000197916.99629.eb.

6. Committee on Trauma. Resources for optimal care of the injured patient. Chicago; 2014. Available at: https://www.facs.org/ \%/media/files/quality\%20programs/trauma/vrcresources.ashx.

7. Hameed SM, Schuurman N, Razek T, et al. Access to trauma systems in Canada 7 Trauma 2010;69:1350-61; discussion 1361, doi:10.1097/TA.0b013e3181e751f7.

8. Lawson FL, Schuurman N, Oliver L, et al. Evaluating potential spatial access to trauma center care by severely injured patients. Health Place 2013;19:131-7; doi:10.1016/j. healthplace.2012.10.011.

9. Brisseau G, Avery B. Trauma Registry Report on injury in Nova Scotia. Nova Scotia: Nova Scotia Department of Health; 2010. Available at: http://www.fecst.gouv.qc.ca/fileadmin/ documents/NS_Trauma_Registry_Report_10_1_.pdf.

10. Moran P. Notes on continuous stochastic phenomena. Biometrika 1950;37:17-23; doi:10.2307/2332142.

11. Oliver MA, Webster R. Kriging: a method of interpolation for geographical information systems. Int 7 Geogr Inform Syst 1990;4:313-32; doi:10.1080/02693799008941549.

12. Branas CC, Mackenzie EJ, Williams JC, et al. Access to trauma centers in the United States. $7 A M A$ 2005;293:2626-33.
13. Doumouras AG, Gomez D, Haas B, et al. Comparing methodologies for evaluating emergency medical services ground transport access to time-critical emergency services: a case study using trauma center care. Acad Emerg Med 2012;19:E1099-108; doi:10.1111/j.1553-2712.2012. 01440.x.

14. Braddock M, Lapidus G, Cromley E, et al. Using a geographic information system to understand child pedestrian injury. Am 7 Public Health 1994;84:1158-61; doi:10.2105/AJPH.84.7.1158.

15. Boyle J, Lampkin C. 2007, Motor Vehicle Occupant Safety Survey. Volume 4: Crash Injury and Emergency Medical Services Report; 2008. doi:10.1016/S0196-0644(98) 70265-3.

16. Amram O, Schuurman N, Yanchar NL, et al. Use of geographic information systems to assess the error associated with the use of place of residence in injury research. Inj Epidemiol 2015;2:29. doi:10.1186/s40621-015-0059-y.

17. Noor AM, Amin AA, Gething PW, et al. Modelling distances travelled to government health services in Kenya. Trop Med Int Heal 2006;11:188-96; doi:10.1111/j.13653156.2005.01555.x.

18. Gething PW, Johnson FA, Frempong-Ainguah F, et al. Geographical access to care at birth in Ghana: a barrier to safe motherhood. BMC Public Health 2012;12:991. doi:10.1186/1471-2458-12-991.

19. Zou HX, Yue Y, Li QQ, et al. An improved distance metric for the interpolation of link-based traffic data using kriging: a case study of a large-scale urban road network. Int 7 Geogr Inform Sci 2012;26:667-89; doi:10.1080/13658816.2011.609488.

20. Miura H. A study of travel time prediction using universal kriging. TOP 2009;18:257-70; doi:10.1007/s11750-009-0103-6.

21. Harmsen AMK, Giannakopoulos GF, Moerbeek PR, et al. The influence of prehospital time on trauma patients outcome: a systematic review. Injury 2015;46:602-9; doi:10.1016/j.injury.2015.01.008.

22. Fakhry SM, Trask AL, Waller MA, et al. Management of brain-injured patients by an evidence-based medicine protocol improves outcomes and decreases hospital charges. 7 Trauma 2004;56:492-9; discussion 499-500. 